\title{
Grain Boundary Energy Interpolation for Uranium Dioxide
}

\author{
Jarin French
}

September 2016

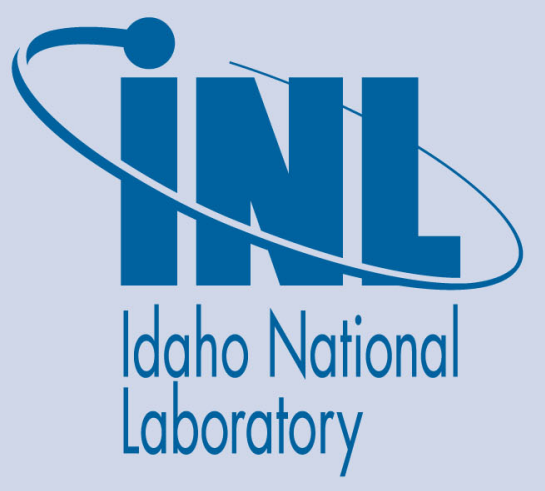

The INL is a U.S. Department of Energy National Laboratory operated by Battelle Energy Alliance 


\title{
Grain Boundary Energy Interpolation for Uranium Dioxide
}

\author{
Jarin French \\ September 2016 \\ Idaho National Laboratory \\ Idaho Falls, Idaho 83415 \\ http://www.inl.gov \\ Prepared for the \\ U.S. Department of Energy \\ Under DOE Idaho Operations Office \\ Contract DE-AC07-05ID14517
}


Brigham Young University - Idaho

VFP Student Research Report

Grain Boundary Energy Interpolation for Uranium Dioxide Jarin French

Mentor: Yongfeng Zhang

Faculty Mentor: Evan Hansen

September 22, 2016 


\begin{abstract}
Grain boundary (GB) energies have an effect on the frequency with which certain GBs are present in a material. Since GBs have a direct impact on a material's properties, knowledge of GB energies is useful in determining those properties. This knowledge is difficult to establish due to an incomplete understanding of the atomic structure of a GB. Previous research has successfully created an interpolation function for the GB energies of face-centered cubic (fcc) metals. An extension of this work was recently done by creating an interpolation function for uranium dioxide $\left(\mathrm{UO}_{2}\right)$, which has a fluorite crystal structure. This work improves the accuracy of that function. Molecular dynamics (MD) simulations were used to calculate more accurate GB energies for $\mathrm{UO}_{2}$, and new fitting parameters were calculated from those results. Comparison with previous work shows an overall improvement.
\end{abstract}




\section{INTRODUCTION}

The effect of GBs is very important in the simulation of materials. Knowledge of the energy at any arbitrary GB allows for more realistic simulations of the microstructure of the material. This allows for accurate calculations of material properties. A challenge in the effort to calculate this information is that GBs and their associated energies are difficult to analytically define. Part of this difficulty stems from the lack of available data for a sufficient number of GBs to get an accurate picture of the GB energy profile. GBs have five degrees of freedom. ${ }^{1-6}$ Thus, the full GB five-space can only be accurately interpolated within through the use of many data points. ${ }^{1}$ In 2014 Bulatov et al. ${ }^{1}$ created a function that accurately interpolates the GB energies of four fcc metals - nickel, aluminum, gold, and copper - with a limited amount of data by taking advantage of the topography of the GB energy hypersurface. With that work they were able to create a "universal" function that describes the GB energies of all fcc metals and depends on only two material-specific parameters. Harbison ${ }^{5}$ implemented the same procedure for $\mathrm{UO}_{2}$, using energies calculated from MD simulations with no anneal. This work uses energies for the same GBs used in Harbison's work, but calculated with an anneal of $800 \mathrm{~K}$.

\section{METHODS}

\section{Literature review}

An in-depth study of Bulatov et al.' ${ }^{1}$ work was conducted to understand their methods for calculating the GB energy parameters. In order to find the energy of an arbitrary GB in the five-space they implemented a hierarchical interpolation method. They started by choosing three three-dimensional (3D) high-symmetry axes to use as scaffolding to build the entire five-dimensional (5D) function. The axes chosen were the $\langle 100\rangle,\langle 110\rangle$, and the $\langle 111\rangle$ sets for their four-, two-, and three-fold rotational symmetries respectively. ${ }^{*}$ Each 3D subset was built from an interpolation of their own one- and two-dimensional subsets. The symmetric tilt and twist GBs for each set were fitted first because of their simplicity. Only the rotation angle is needed to fully define the energies for these subsets, making them one-dimensional (in Fig. 1a, the darker bands in the smaller circles). From the symmetric tilt subset, the asymmetric, or general, tilt subset was interpolated. A second rotation angle defining the rotation of the second grain makes this subset two-dimensional (the lighter, wider band of color around the symmetric subset). A combination of the general tilt subset (two dimensions) and the twist subset (one dimension) was used to interpolate the threedimensional subset for each high-symmetry axis (the three smaller circles). These three three-dimensional subsets were then used to interpolate the GB 5D space.

Part of the development of this 5D function was accomplished through visual representations of the GB space. However, representing GBs is difficult because of their five degrees of freedom. Different methods have been developed to represent them, each with their advan-

${ }^{*}$ For cubic crystals, rotations of $90^{\circ}, 180^{\circ}$, or $120^{\circ}$ about any $\langle 100\rangle,\langle 110\rangle$, or $\langle 111\rangle$ axis respectively is a symmetry operation. Thus, the $\langle 100\rangle$ set is four-fold symmetric $\left(360^{\circ} / 90^{\circ}=4\right)$, the $\langle 110\rangle$ set is two-fold symmetric $\left(360^{\circ} / 180^{\circ}=2\right)$, and the $\langle 111\rangle$ set is three-fold symmetric $\left(360^{\circ} / 120^{\circ}=3\right)$. 


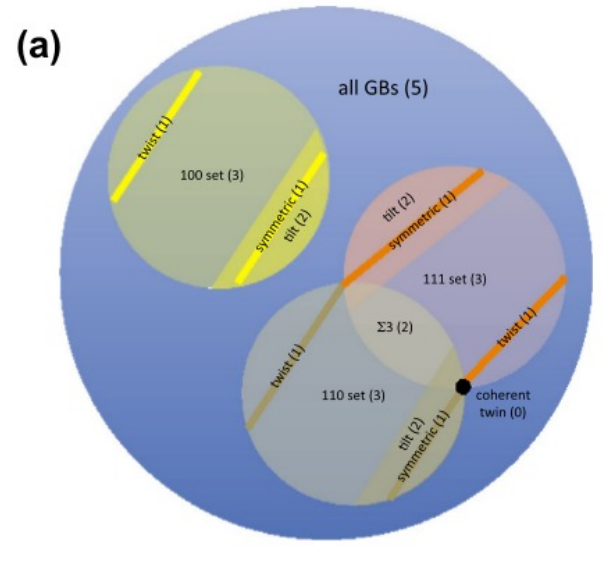

(a)

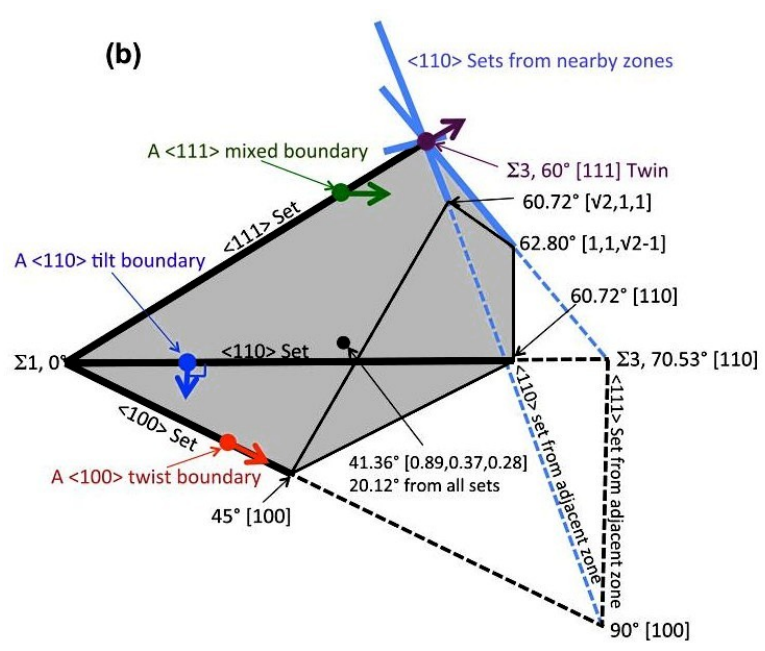

(b)

FIG. 1: Fig. 2 from Bulatov et al. ${ }^{1}$ (a) demonstrates the theoretical relationship between the high-symmetry subsets of the 5D GB space. Each multi-dimensional subset is interpolated from smaller-dimensional subsets. (b) shows the Rodrigues space representation of the fundamental zone of all GBs as built from three high-symmetry axes. The unit vectors along the axes identify the boundary plane inclination. A parallel vector thus represents a twist boundary, a perpendicular vector represents a tilt boundary, and a vector that is neither parallel nor perpendicular represents a mixed boundary.

tages and disadvantages. One method is to use Rodrigues vectors to represent rotations in Rodrigues space. This space utilizes concepts from an axis-angle representation, where the axis of misorientation ${ }^{\dagger}$ specifies the point in space, and the angle ${ }^{\ddagger}$ determines the magnitude of the vector. In Rodrigues space, the magnitude of the vector is defined as $\tan (\theta / 2)$. Thus, a Rodrigues vector $(\boldsymbol{R})$ is defined by an axis $\left(\boldsymbol{a}\right.$, where $\boldsymbol{a}$ has components $a_{x}, a_{y}$, and $\left.a_{z}\right)$ and an angle $(\theta)$ by, ${ }^{8-12}$

$$
\boldsymbol{R}=\boldsymbol{a} \tan \left(\frac{\theta}{2}\right) .
$$

This specifies only three of the five degrees of freedom. The other two are represented in Fig. 1b by a unit vector at the point along the axis. A parallel vector represents a twist boundary, and a perpendicular vector represents a tilt boundary. Anything else represents a mix of twist and tilt (or a mixed boundary). One difficulty in using Rodrigues space is that it is an infinite space, as it simply maps an axis and an angle onto a Cartesian coordinate system. ${ }^{10,13}$ Every crystal has some sort of symmetry, ${ }^{14}$ and it is by taking advantage of these crystal symmetries that the infinite space, and the 5D GB space can be

\footnotetext{
${ }^{\dagger}$ The misorientation of a grain describes how one grain is oriented with respect to another. A perfect crystal has no misorientation. There are two main types of misorientation, twist and tilt, which indicate an axis of misorientation perpendicular or parallel to the GB normal respectively. Twist boundaries are made up of screw dislocations, and tilt boundaries are made up of edge dislocations. ${ }^{7}$

$\ddagger$ The angle here specifies the amount by which the grain is rotated to get to its current orientation, and is measured from a lab frame. The lab frame is an arbitrary frame from which the researcher makes the measurement.
} 
greatly simplified..$^{1,2,4,8,15}$ This simplification comes in the form of a "fundamental zone" where every GB is represented in a compact space that is conceptually easier to visualize. The fundamental zone represents all GBs in that every point within the fundamental zone is unique, and every point outside of the fundamental zone can be represented by a point inside the fundamental zone. ${ }^{8-10}$ Bulatov et al. ${ }^{1}$ utilized this idea to create Fig. $1 \mathrm{~b}$, and also as a basis for their interpolation function.

\section{The fitting}

Part of the difficulty that comes with creating an interpolation function for GB energy is the lack of descriptive functions describing what happens at "high-angle boundaries." 3 At these GBs different dislocations ${ }^{\S}$ begin to overlap making it hard to a) distinguish the individual contributions to the overall lattice imperfection, and b) determine a functional form of the GB energies. ${ }^{3,6}$ Wolf ${ }^{16}$ created a model that successfully predicted GB energies for these high-angle boundaries. He built off of work done by Read and Shockley ${ }^{17}$ who modeled GB energies at low-angle boundaries. Bulatov et al. ${ }^{1}$ used the resultant ReadShockley-Wolf (RSW) model as the basis for their interpolation functions.

In 2015 Harbison $^{5}$ applied Bulatov et al.'s methods to $\mathrm{UO}_{2}$. He fit $158 \mathrm{~GB}$ energies, ${ }^{5}$ calculated by MD simulations, to Bulatov et al.'s interpolation function using a grid-search algorithm. From this he obtained the 43 parameters necessary to calculate any arbitrary GB energy. This work used the same GBs in Harbison's work, but calculated their energies with an $800 \mathrm{~K}$ anneal to obtain a better fit. By running the MD simulations with that anneal, most of the GB energies were found to be lower and thus produced a more accurate fit for the interpolation function.

The fitting procedure is generally the same for all nine data subsets. First, the parameters relating to the set $\mathbb{I}$ are read in from previous fittings (in the case of no previous runs, the copper parameters that were calculated by Bulatov et al. ${ }^{1}$ are used). Important angles are specified where low energies are expected, such as a $\Sigma 5$ boundary for the $\langle 100\rangle$ symmetric tilt subset. These angles are calculated based on coincident site lattice (CSL) theory, which states that the more lattice points that coincide, the lower the energy. ${ }^{3,6}$ Each energy in the parameter-vector is listed as a scaled value based on the $e_{R G B}$ parameter - this is a parameter that represents the energy of an arbitrary, random GB, and can be seen as an average of the GB energies. Thus, to make relevant comparisons, the energies are unscaled. An initial step size is then set (various step sizes were needed for the different sets to avoid numerical problems), and data for the specific subsets is read in from a database. The misorientation angles and their associated energies are sorted in order from the smallest to the largest angle, then all of the parameters and the angle-energy pairs are passed into a grid-search fitting function.

Once the nine subsets are fitted, the mixing and weighting parameters that define the relationship between the subsets are calculated in a similar manner to determine the full 5D function. To test the function, results will be compared to simulation results for the $\langle 210\rangle$

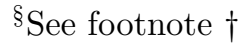

${ }^{\mathbb{I} B u l a t o v}$ et al. ${ }^{1}$ identifies a set of functions for each subset. Each of these functions requires different parameters which must be fit to data. These are then used to form a vector containing all of the parameters for each subset which are used to fit to the entire $5 \mathrm{D}$ space.
} 
set. Fitting results of the six one-dimensional subsets are provided in the following section. For a list of the parameters calculated from the fitting procedure, see Table 1.

\section{RESULTS AND DISCUSSION}

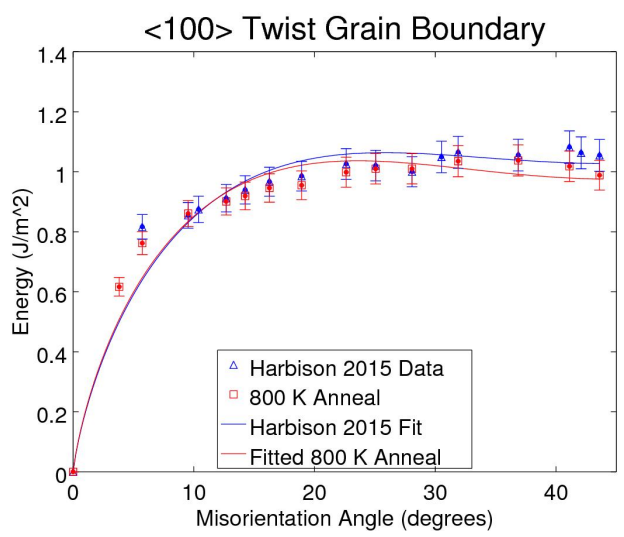

(a)

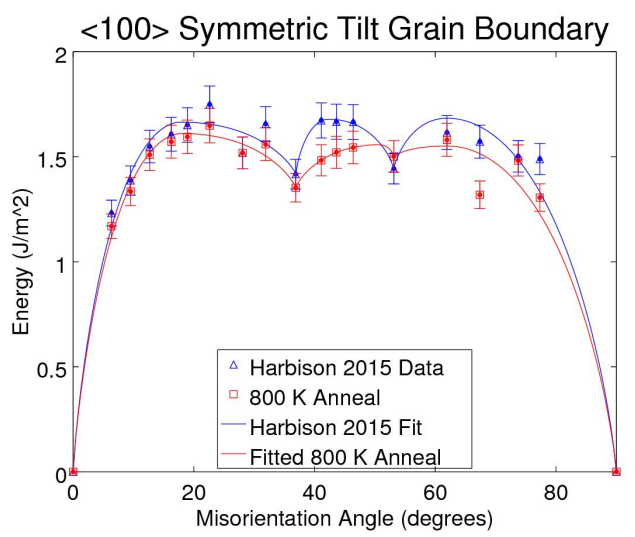

(b)

FIG. 2: The $\langle 100\rangle$ twist (a) and tilt (b) results. In general the re-calculated energies are lower, with significant differences around $40^{\circ}$ to $50^{\circ}$ in the tilt subset. The positive concavity in the twist subset around $40^{\circ}$ is unexpected, and may indicate the presence of a missing cusp. There is a possible cusp around $30^{\circ}$ in the twist subset, and around $68^{\circ}$ in the tilt subset.

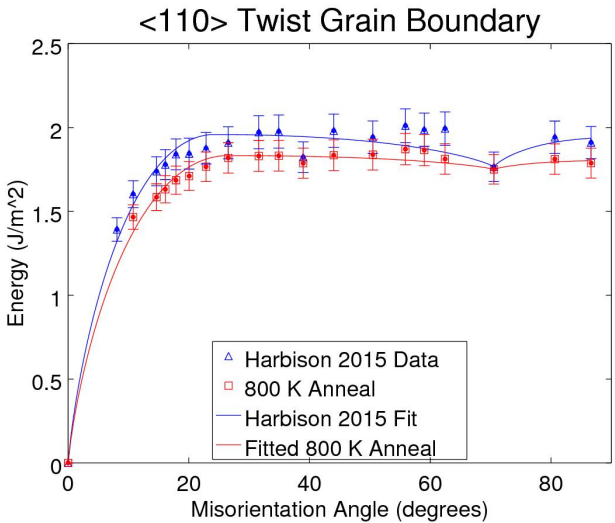

(a)

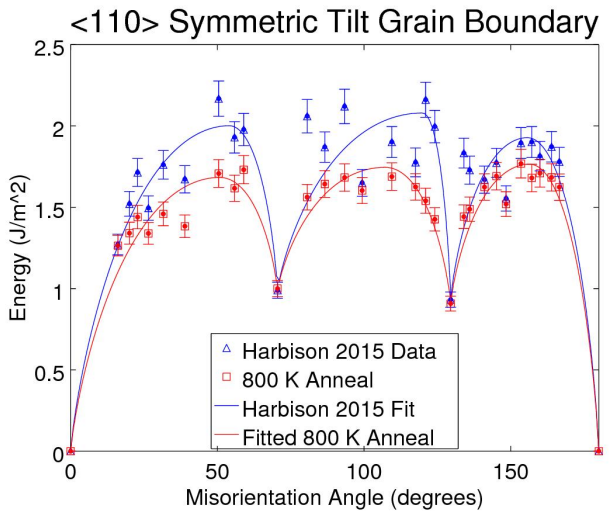

(b)

FIG. 3: The $\langle 110\rangle$ twist (a) and tilt (b) results. Significant decreases in energy are found for both subsets. Possible cusp locations are around $40^{\circ}$ in the twist subset, and around $40^{\circ}$, $90^{\circ}$, and $140^{\circ}$ in the tilt subset.

Figs. 2 to 4 show a comparison of this work's results with results from Harbison ${ }^{5}$ for the one-dimensional subsets as outlined by Bulatov et al. ${ }^{1}$ Initial MD calculations of the $\langle 100\rangle$ symmetric tilt GB energies using the $800 \mathrm{~K}$ anneal (Fig. 2b) showed a deep cusp around 


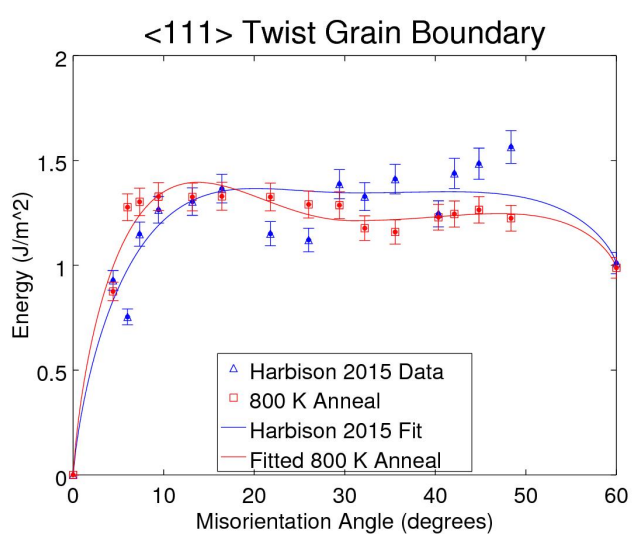

(a)

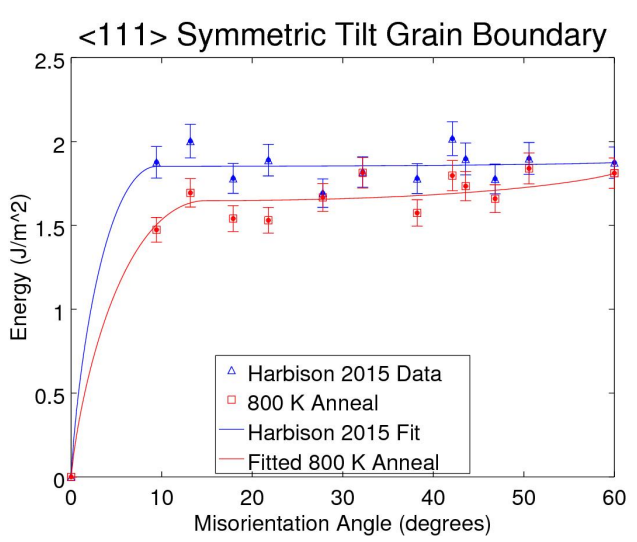

(b)

FIG. 4: The $\langle 111\rangle$ twist (a) and tilt (b) results. Some energies are found to be lower, but some are also found to be higher. The positive concavity present in these results is unexpected, and could indicate the presence of cusps. A possible location for the twist subset is around $33^{\circ}$. Additional data is needed to determine possible cusp locations for the tilt subset.

$28^{\circ}$ which was unexpected. An analysis of the MD simulation results for this misorientation revealed that, in this case, the two crystals had re-aligned, changing the misorientation angle. This realignment caused the GB energy to be much lower than expected. Comparison with the simulation result used by Harbison revealed that his crystal structure did not realign. While Harbison's data was not calculated with the anneal and thus may not represent a global minimum, we feel justified in using his results for the energy at $28^{\circ}$ as it follows the trend that the data around it sets.

Of the symmetric tilt GB energy sets, the $\langle 110\rangle$ set has the most improvement. All three sets showed a general decrease in the energy, giving us confidence that we have achieved a better fit that more accurately describes $\mathrm{GB}$ energies in $\mathrm{UO}_{2}$. Each of these sets provides more opportunity for research however. The $\langle 100\rangle$ set needs more work done for data points after around $50^{\circ}$. The scatter associated with those points seems to be higher, and the possibility of a slight cusp presents itself around $68^{\circ}$. The $\langle 110\rangle$ set as mentioned shows the most improvement, but there are some low points in the second and third "humps" that do not follow the trend, indicating further possibility for cusps. The first part of the function (the first hump) needs additional data to determine the possibility of a cusp between $40^{\circ}$ and $50^{\circ}$. The fitted curve to the $\langle 111\rangle$ set now has an upward trend that is unexpected. The scatter associated with these data points is also relatively high, leading to the possibility of a completely different set of functions to define this subset.

The twist GB energy sets vary in their success. The $\langle 100\rangle$ set shows very little difference between Harbison's ${ }^{5}$ work and this work. There is a slight positive concavity at the end of the fitting for this subset, and this is unexpected, indicating the possibility of a cusp. This cusp may occur around $30^{\circ}$. The $\langle 110\rangle$ set has a definite decrease in the overall energies, creating a plateau profile. An additional cusp around $40^{\circ}$ is being considered. The $\langle 111\rangle$ set has the least improvement. From the work done by Bulatov et al. ${ }^{1}$ this work expected to see a plateau as demonstrated by Harbison's ${ }^{5}$ fitting. Instead, the fitting produced a curved 
energy profile, indicating the potential for at least one cusp. A possible location of this cusp is around $35^{\circ}$.

\section{CONCLUSION}

This work aimed to create a more accurate interpolation function for $\mathrm{GB}$ energies in $\mathrm{UO}_{2}$. Results in general showed a decrease in energy, which was expected. However, each energy did not decrease the same amount, revealing additional characteristics of the energy profiles. Future work will focus on quantifying those characteristics in an effort to more accurately interpolate any arbitrary GB energy in $\mathrm{UO}_{2}$. Further analysis in this direction will likely lead to a different set of functions, increasing the number of parameters necessary to define the 5D GB energy space for $\mathrm{UO}_{2}$. Additional efforts can be directed towards analyzing the GB energy profiles of other fluorite-crystal-structured materials to determine a general or universal interpolation function for that crystallographic class. 


\section{ACKNOWLEDGMENTS}

I would first like to thank the Department of Energy Office of Science for the Visiting Faculty Program (VFP) - Student, and the Nuclear Energy Advanced Modeling and Simulation (NEAMS) program which allowed me this opportunity. Thanks also goes to Brigham Young University - Idaho and Idaho National Laboratory for providing me with the necessary facilities to do this work. I would especially like to thank Yongfeng Zhang for his patience and guidance as I have worked on this research. Additionally I would like to thank Evan Hansen, John-Michael Bradley, and Xianming Bai for their valuable contributions to my understanding. Finally, I would like to thank my wife who has been so supportive of me as I have spent so much time doing this work, and who has been a constant source of strength to me. 


\section{References}

${ }^{1}$ V. V. Bulatov, B. W. Reed, and M. Kumar, Acta Mater. 65, 161 (2014).

${ }^{2}$ S. Patala and C. A. Schuh, Philosophical Magazine 93, 524 (2013).

${ }^{3}$ P. Lejček, Grain Boundaries: Description, Structure and Thermodynamics, pages 5-24, Springer Berlin Heidelberg, Berlin, Heidelberg, 2010.

${ }^{4}$ E. R. Homer, S. Patala, and J. L. Priedeman, Sci. Rep. 5, 15476 (2015).

${ }^{5}$ T. Harbison, Anisotropic grain boundary energy function for uranium dioxide, B.S. Thesis, Brigham Young University - Idaho, 2015.

${ }^{6}$ G. S. Rohrer, J. Mater. Sci. 46, 5881 (2011).

${ }^{7}$ W. D. Callister Jr., Materials Science and Engineering: An Introduction, John Wiley \& Sons Inc, USA, 2003.

${ }^{8}$ A. Morawiec and D. P. Field, Phil. Mag. A 73, 1113 (1996).

${ }^{9}$ R. Becker and S. Panchanadeeswaran, Textures and Microstructures 10, 167 (1989).

${ }^{10}$ F. C. Frank, Metallurgical Transactions A 19A, 403 (1988).

${ }^{11} \mathrm{~V}$. Randle and O. Engler, Introduction to Texture Analysis: Macrotexture, Microtexture and Orientation Mapping, CRC Press, USA, 2000.

${ }^{12}$ L. Priester, Geometrical Order of Grain Boundaries, pages 3-28, Springer Netherlands, Dordrecht, 2013.

${ }^{13}$ D. M. Kirch, Fundamentals of grain boundaries and triple junctions, pages 1-7, Cuvillier, Göttingen, 2008.

${ }^{14}$ H. T. Stokes, Solid State Physics: For advanced undergraduate students, BYU Academic Publishing, Provo, Utah, 2007.

${ }^{15}$ S. Patala, J. K. Mason, and C. A. Schuh, Progress in Materials Science 57, 1383 (2012).

${ }^{16}$ D. Wolf, Scripta Metallurgica 23, 1713 (1989).

${ }^{17}$ W. T. Read and W. Shockley, Physical Review 78, 275 (1950). 
Table 1: This table gives the parameters for $\mathrm{UO}_{2}$ that generate the energy function.

\begin{tabular}{rll}
\hline \hline Array number & Parameter name & Parameter value \\
\hline 1 & Energy Scaling Factor $\left(e_{R G B}\right)$ & $1.615789 \mathrm{~J} / \mathrm{m}^{2}$ \\
2 & $\langle 100\rangle$ Max Distance & 0.405 \\
3 & $\langle 110\rangle$ Max Distance & 0.739 \\
4 & $\langle 111\rangle$ Max Distance & 0.352 \\
5 & $\langle 100\rangle$ Weight & 16.2 \\
6 & $\langle 110\rangle$ Weight & 1.65 \\
7 & $\langle 111\rangle$ Weight & 0.08 \\
8 & $\langle 100\rangle$ Tilt/Twist Mix Power Law (1) & 0.03325 \\
9 & $\langle 100\rangle$ Tilt/Twist Mix Power Law $(2)$ & 0.00053 \\
10 & Maximum $\langle 100\rangle$ Twist Energy & 0.60903 \\
11 & $\langle 100\rangle$ Twist Shape Factor & 1.44857 \\
12 & $\langle 100\rangle$ Asymmetric Tilt Interpolation Power & 35.6 \\
13 & $\langle 100\rangle$ Symmetric Tilt First Peak Energy & 1.00578 \\
14 & $\langle 100\rangle$ Symmetric Tilt First 55 Energy & 0.84456 \\
15 & $\langle 100\rangle$ Symmetric Tilt Second Peak Energy & 0.97259 \\
16 & $\langle 100\rangle$ Symmetric Tilt Second $\Sigma 5$ Energy & 0.9379 \\
17 & $\langle 100\rangle$ Symmetric Tilt $\Sigma 17$ Energy & 0.96881 \\
18 & $\langle 100\rangle$ Symmetric Tilt First Peak Angle & 0.31569 \\
19 & $\langle 100\rangle$ Symmetric Tilt Second Peak Angle & 0.88538 \\
20 & $\langle 110\rangle$ Tilt/Twist Mix Power Law (1) & 1.05731 \\
21 & $\langle 110\rangle$ Tilt/Twist Mix Power Law $(2)$ & 1.57844 \\
22 & $\langle 110\rangle$ Twist Peak Angle & 0.46145 \\
23 & $\langle 110\rangle$ Twist Peak Energy & 1.14442 \\
24 & $\langle 110\rangle$ Twist $\Sigma 3$ Energy & 1.09306 \\
25 & $\langle 110\rangle$ Twist 90 Energy & 1.12621 \\
26 & $\langle 110\rangle$ Asymmetric Tilt Shape Factor & 3.18431 \\
27 & $\langle 110\rangle$ Symmetric Tilt Third Peak Energy & 1.05139 \\
28 & $\langle 110\rangle$ Symmetric Tilt $\Sigma 3$ Energy & 0.61703 \\
29 & $\langle 110\rangle$ Symmetric Tilt Second Peak Energy & 1.09024 \\
30 & $\langle 110\rangle$ Symmetric Tilt $\Sigma 11$ Energy & 0.56686 \\
31 & $\langle 110\rangle$ Symmetric Tilt First Peak Energy & 1.10238 \\
32 & $\langle 110\rangle$ Symmetric Tilt Third Peak Angle & 0.88736 \\
33 & $\langle 110\rangle$ Symmetric Tilt Second Peak Angle & 1.87115 \\
34 & $\langle 110\rangle$ Symmetric Tilt First Peak Angle & 2.73099 \\
35 & $\langle 111\rangle$ Tilt-Twist Linear Interpolation & 5.90062 \\
36 & $\langle 111\rangle$ Twist Shape Factor & 1.78856 \\
37 & $\langle 111\rangle$ Twist Peak Angle & 0.5398 \\
38 & $\langle 111\rangle$ Twist Peak Energy & 0.75736 \\
39 & $\langle 111\rangle$ Symmetric Tilt Peak Angle & 0.25966 \\
40 & $\langle 111\rangle$ Symmetric Tilt Max Energy & 1.02884 \\
\hline & &
\end{tabular}

Continued on next page. 
Table 1 - Continued from previous page

\begin{tabular}{rll}
\hline Array number & Parameter name & Parameter value \\
\hline 41 & $\langle 111\rangle$ Symmetric Tilt $\Sigma 3$ Energy & 1.13114 \\
42 & $\langle 111\rangle$ Asymmetric Tilt Symmetry Point Energy & 3.18623 \\
43 & $\langle 111\rangle$ Asymmetric Tilt Scale Factor & 0.0706 \\
\hline \hline
\end{tabular}

